\title{
Target Professions Based Approach for Individual Learning Pathway Creation
}

\author{
Anton Govorov, Anastasiia Chernysheva, Svetlana Derkunskaia, Valeriya Artamonova, Carina \\ Babayants, and Sergei Koriakov
}

\begin{abstract}
University education is becoming more student-centered and personalized in recent years, whereas connections between professional skills and learning outcomes are recognized vaguely. The article proposes an approach to create relations between skills that students acquire in a higher education process and those required in various careers. The approach makes it possible to trace consistency between course material, educational programs, and professions. Thus, the implementation of skills and interdisciplinary connections allows to build the most relevant individual learning tracks for a specific career and provides students with valuable recommendations. The proposed approach is implemented in ITMO University's (Russia) web service "Educational Program Maker," initially designed for education process management. The system ensures the development of educational program elements, provides a platform for describing relations between educational entities, and enables users to choose a curriculum for a specific profession.
\end{abstract}

Index Terms - Graph model, individual learning pathway, professional skills, web development.

\section{INTRODUCTION}

Today, numerous fields of knowledge develop promptly. Consequently, skills required in different industries become irrelevant quickly. For example, at the beginning of the $21 \mathrm{st}$ century, there has been significant growth in information technology. New solutions have easily outdone the ones that were irreplaceable a decade ago. This process cannot be changed.

The development of information technology has made it possible to expand the range of options in educational process organizing. Various educational tools that can improve the quality of education have appeared. These tools include electronic simulators, learning management systems, and online courses.

Higher education systems strive to be as student-centered as possible, whereas connections between professional skills and learning outcomes are recognized vaguely. It is necessary to implement mechanisms that allow monitoring the consistency of knowledge between professions and educational programs, and therefore solving the problems of learning analytics is becoming increasingly relevant. Individual learning pathway creation is one of these tasks.

This paper outlines an approach to describing syllabi and

Manuscript received May 27, 2021; revised June 30, 2021

The authors are with the Faculty of Infocommunication Technologies, ITMO University, Saint Petersburg, Russia (corresponding author: A. Chernysheva; e-mail: avchernysheva@itmo.ru). professions that traces the content consistency between academic courses, educational programs, and professions. The current approach is based on another one proposed in [1]. There, the educational process consists of educational entities described by keywords. One set of keywords can define the educational plan's academic courses, and the other one represents the necessary professional skills. These sets' inter-section shows which disciplines are most likely to lead to acquiring the skills necessary for the chosen profession. Thus, a multilayer model of the educational process is created, where a corresponding keyword graph represents each layer. Connections between layers are determined by matching keywords, hierarchical, or prerequisite relations. Multilayer model exploration would allow building individual learning pathways within the educational program and recommend them.

The paper is composed as follows: section two introduces related work in the field of curriculum design, personalization, and evaluation, section three provides a detailed discussion of the methods for individual learning pathway creation, while section four presents the implementation of the proposed approach. We conclude with an overview of the approach implementation and an outline for future work.

\section{RELATED WORK}

The dynamic development of modern society has affected all aspects of human life, including the education sector. Technologies develop rapidly, which entails ongoing knowledge maintenance through skill improvement and retraining. The student's ability to learn independently and build individual learning pathways that can contribute to professional development throughout life is vital [2]. The problem of curriculum and syllabus design has been studied thoroughly worldwide.

There are various studies and methodological guidelines related to curriculum creation. Kharitonov I. [3] says that according to the leading higher education goal, it is the professional activity that sets and determines the goals of studying all academic courses - the content and structure of the corresponding learning activities for students. At the same time, he notes that the existing methods for learning process management based on the formalized approach to constructing academic courses are insufficiently developed.

Jones N. [4] examines how the layout of syllabi can aid or tangle academic course content that is vital to learner success. The study proposes considering students as expert end-users with valuable knowledge proper to syllabi creation using a 
human-centered design approach.

Wette R. [5] states that teachers are "curriculum makers and adapters rather than mere transmitters of written plans and prescriptions." Syllabi are constantly being revised in response to the powerful influence of student feedback and curriculum restrictions.

Dubicki E. [6] reviews curricular learning outcomes, assignments, library resources, and services references. Then the researcher aligns them with information literacy and proposes an approach to information literacy instruction, with novice skills introduced in lower-level courses and expert skills in upper-level and graduate courses to meet learning outcomes.

Several studies highlight the importance of education for students' future careers. Sharov A. [7] and Sokolova A. [8] consider university education's role in building professional careers. Sharov says that a university program should combine theoretical concepts and efficient ways to apply them in practice. Sokolova A.S. concludes in her work that the curriculum should focus on the context of professional issues and consider student's learning pathways. Thus, to avoid a mismatch between the labor market demand and graduates' formed competencies, a student's mastery of specific skills at university should be based on the competencies required to build a professional career.

Some researchers have also studied connections between career, learning outcomes, professional skills, and the relationship between career training and employee productivity. Khailova L. [9] addresses curriculum mapping to improve curricular cohesiveness, scaffolding, and goal achievement. As a result of approach implementation, students' learning objectives and outcomes are matched and used to support the educational process.

Woolley et al. [10] conclude that learning outcomes and academic performance are improved when studying academic courses that match their interests and aptitudes. Therefore, it is essential to guide students to career choices following their interests and abilities.

Farah Haneef et al. [11] suggest using network science to understand the relations between academic courses, student studies, and the impact of those subjects on their careers. Their model helps to understand the positive and negative effects of specific academic courses within the curriculum and graduates' careers.

Nowadays, individual learning is one of the main trends in higher education, although it has several disadvantages. Yurlovskaya I.A. [12] reveals the essential characteristics of individualized learning, provides justifications for terms and conditions of its implementation in universities. The author also lists basic principles of establishing personal and professional student development conditions and clarifies teacher and student roles. The effectiveness of individualized learning depends crucially on the natural combination of its two aspects: high-quality teaching activity and student activity stimulation.

Fewer studies focus on curricula and syllabi evaluation.

Gregori-Giralt E. and Menéndez-Varela J.-L. [13] note that "a growing trend among universities to promote systems of curricula and academic course evaluation entails more responsibility for faculties and departments." There is a noticeable gap in the research on curricula evaluation concerning their use and validation. Authors examine the content aspect of validity in a rubric-based assessment system for course syllabi.

The current study proposes a learner-centered approach of individual curriculum creation based on students' future professions.

\section{MethodS}

A significant number of educational entities describe the education process. First, it would be appropriate to introduce an educational entity concept. An educational entity is an educational program element connected by structure or logic relations. Educational entities may be structured or unstructured, whether structured or unstructured data signify them. Unstructured educational entities are subject areas. They may be described by keywords and represent the curriculum components' conceptual basis. Structured educational entities result from the educational program's hierarchy, contents, and requirements. They are syllabi, curricula, general characteristics, academic schedule, and individual learning pathways derived from a specific curriculum. These entities may be time-dependent or time-independent, whether they are or are not supposed to be modified according to the real-time updates. There may be diverse approaches to individual learning pathway creation based on the given classification.

The current study adds a noneducational entity which is the target profession. Each entity, including target professions, may be described by a set of keywords extracted from the educational entities' descriptions. Thereby data preparation includes three steps:

1) Keyword graph creation and labeling.

2) Educational program processing.

3) Professional skills extraction and processing.

\section{A. Data Preparation}

Keyword graph creation and labelling. Describing a subject area or an educational entity by a set of keywords is an approach proposed earlier in [14]. Testing proved that the methodology is a convenient solution for any research area to determine its structure, components, and closely related domains.

First, we applied the developed approach to the syllabus analysis and discussed a two-layer graph model. On the first layer, there are curriculum courses; on the second one, there are keywords extracted from section titles and topics within the academic courses. Relations between keywords define relations between courses, allowing us to build learning pathways within a curriculum. The proposed model is depicted in Fig. 1.

Individual learning pathway creation implies the necessity of another layer representing the curriculum. Thus, the model becomes three-layer, and the connections are built by the same logic as in the two-layer one. Relations between academic courses and curricula are defined by the academic course's presence in the curriculum. The three-layer model is presented in Fig. 2. Furthermore, the model can be completed with other educational entity classification layers. 


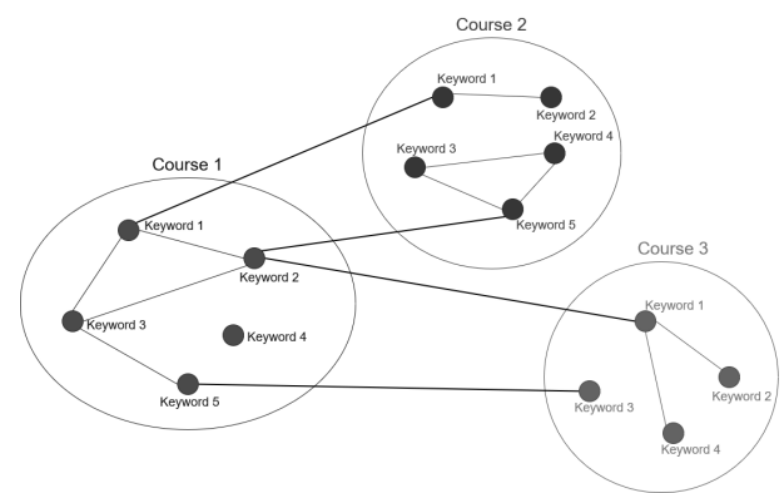

Fig. 1. Two-layer graph model.

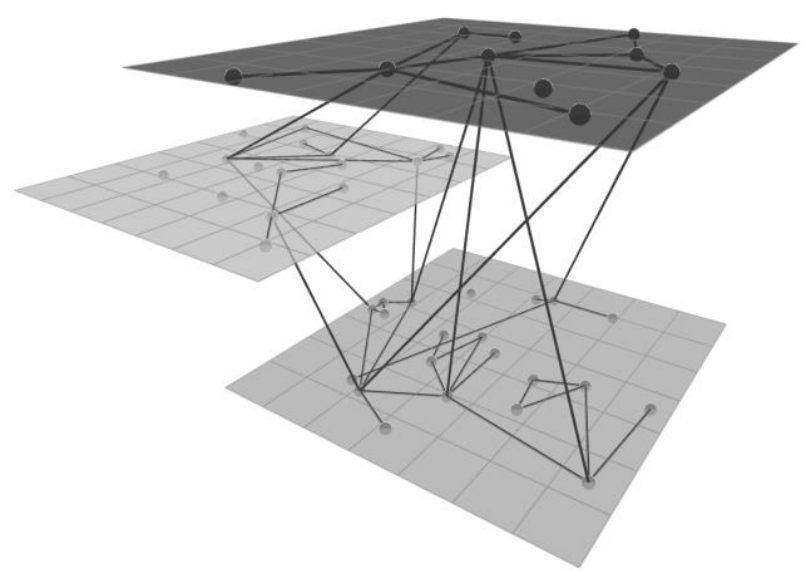

Fig. 2. Three-layer graph model.

It is principal to label the relations between academic courses' basis-level keywords to analyze and interpret educational entities' relations. There are three types of relations distinguished between keywords:

1) Hierarchy $(\subset)$ describes the higher-level educational entity structure in terms of keywords. A hierarchical relation produces two subtypes of relations: "includes" ("one-to-many") and "are parts of one section" ("many-to-many").

2) Prerequisite $(\rightarrow)$ type of connection explains a logical sequence of keywords. One keyword may have many prerequisites, and at the same time, can be a prerequisite for a set of keywords itself ("many-to-many").

3) Identity ( $\equiv$ ) is a type of connection between keywords that describe synonymous entities alongside equivalents in other languages ("many-to-many").

Algorithm 1 illustrates the keyword graph creation and labeling process.

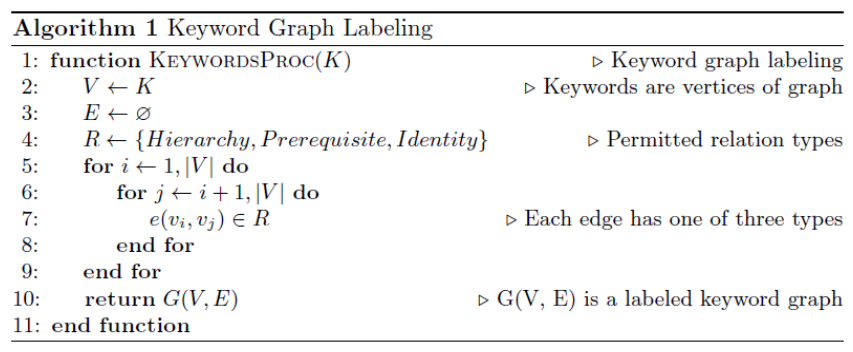

Educational program processing. After keywords graph labeling, educational programs are being processed. Processing includes two substeps. First, academic courses get a selectivity type label. Each of them may be compulsory or optional due to the curriculum module type. Then, each course is described by a set of keywords, as discussed in the previous section. Algorithm 2 demonstrates curricula processing.

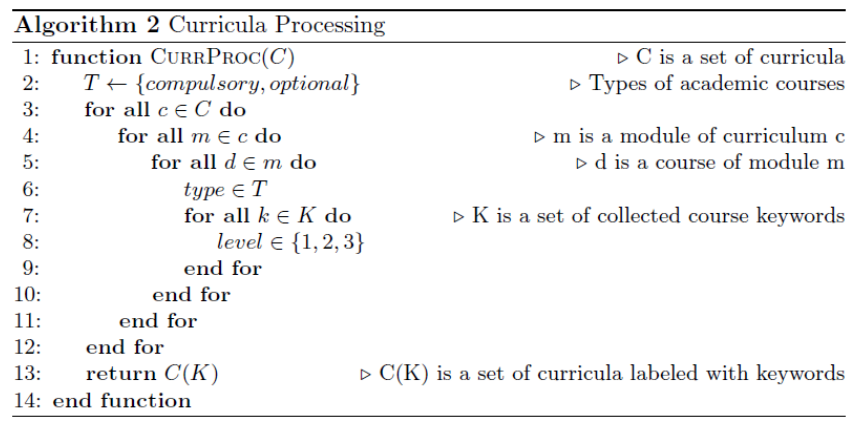

Professional skills extraction and processing. Vacancy aggregators provide the most relevant information on a specific career's professional skills. The first and the most crucial step is to choose an aggregator with the highest precision of recommendations in a domain. Then professional skills are extracted for a chosen career and sorted in descending order of frequency. Each collected skill or, simplistically, a keyword can be classified as a core, additional, or noise skill by its frequency. Although experts can set the threshold according to the domain, testing showed that a balanced threshold is $20 \%$ of vacancies requiring a particular skill for core skills and $1 \%$ for noise. The rest keywords are considered additional. Algorithm 3 shows the pipeline of professional skills processing.
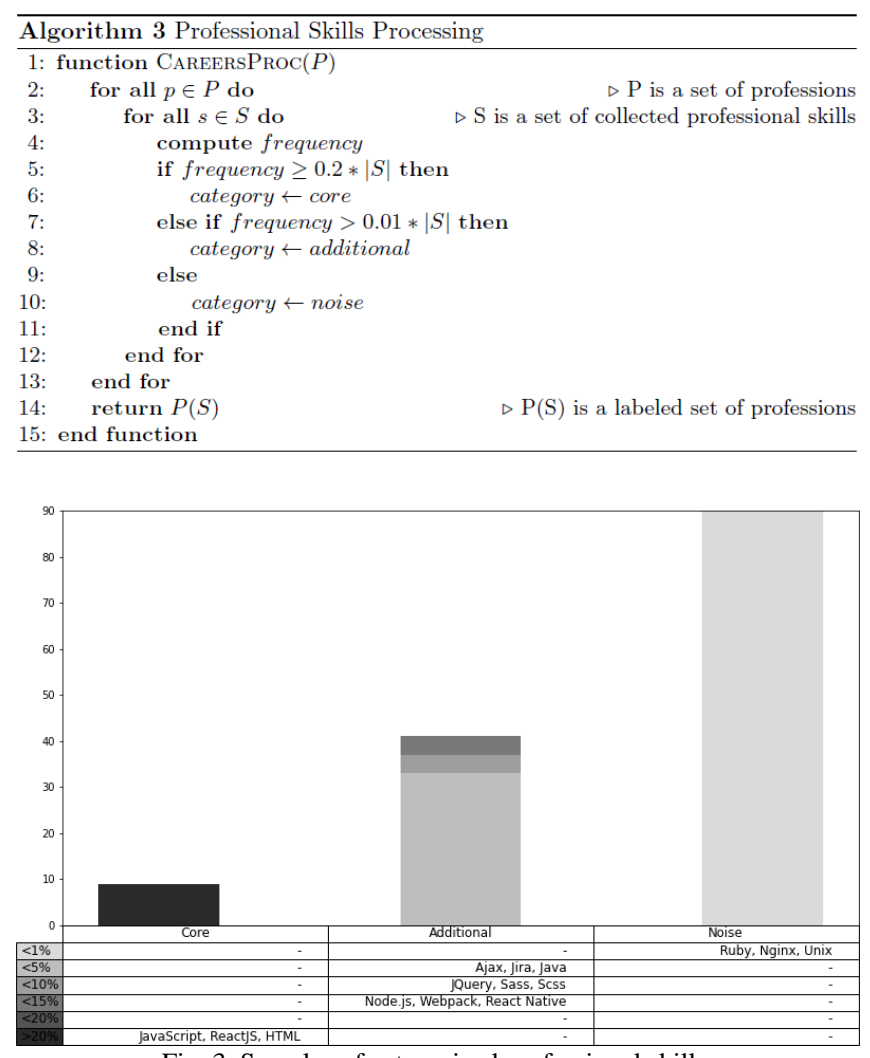

Fig. 3. Samples of categorized professional skills.

For example, 306 vacancies for a Front-End Developer were collected from [15], and frequencies for 140 skills were 
obtained. Then, this list has been cleared from "noise." After eliminating the noise, a set of 50 skills was left. Core skills found in more than $20 \%$ of vacancies were highlighted from this set. The remaining skills were classified as additional. Fig. 3 displays the sample characteristics of Front-End Developer data.

\section{B. Educational Program Recommendation}

There may be dozens or hundreds of possible learning tracks within a particular curriculum, considering the optional academic courses. In such terms, a recommendation of an educational program or individual learning pathway is a ranked list of educational programs based on the user's career choice. The list of recommendations has to meet the following conditions:

1) Educational programs correspond to the level of education.

2) Educational programs correspond to the user's year of admission to the university.

3) Educational programs in the output results are sorted descending order by the $P$-score.

$P$-score is a metric that considers an educational program's compliance ratio with the chosen profession. $P$-score can be calculated for any educational program if the input data is available. When a user chooses a profession, the system prepares two professional skill lists: core skills and additional skills. These two lists are the input data for $P$-score.

$P$-score varies between 0 and 1 . If an educational program is wholly divorced from the chosen career, $P$-score equals 0 . If $P$-score equals 1 , the educational program is entirely consistent with the profession.

On the one hand, $P$-score should consider the core and additional skills coverage ratio of the curriculum courses; on the other hand, it should reflect the curriculum courses' compliance ratio with the chosen profession's core and additional skills. Thereby $P$-score (1) is a harmonic mean of two coefficients $C o v$ and Foc, both varying from 0 to 1 .

$$
P=\frac{2 \times(\operatorname{Cov} \times F o c)}{\operatorname{Cov}+F o c}
$$

Professional skills coverage ratio by curriculum courses (Cov) is a proportion of professional skills that emerge through subject mastery. Both core and additional skills are considered (2).

$$
\text { Cov }=0.8 \times \text { Core }+0.2 \times \text { Additional }
$$

Educational programs focus on developing a competency ratio $(F o c)$ is a proportion of curriculum courses that form professional skills.

In the balanced $P$-score formula (3), a parameter is a non-negative number that allows to consider the contribution of coverage and focus ratios with different weights. For $=1$, the balanced metric is reduced to (1). If it is necessary to prioritize the coverage ratio, then varies from 0 to 1 . If focus is a higher priority, then the parameter must be strictly greater than 1 .

$$
P=\frac{\left(\alpha^{2}+1\right) \times(\operatorname{Cov} \times F o c)}{\alpha^{2} \times \operatorname{Cov}+F o c}
$$

It is necessary to consider all possible individual curricula that can be formed based on the educational program curriculum to calculate coverage and focus ratios. Further calculations should be performed on the individual plan version (or versions) with these ratios' maximum values.

If a set of keywords for a compulsory academic course contains at least one professional skill, then the course is suitable. If at least one academic course from a module of optional courses contains at least one necessary professional skill, then the course is also suitable. The number of suitable academic courses needs to be counted for each specialization; the specialization with the most considerable number of appropriate courses must be chosen. Electives are not considered while calculating the metric. The calculated value is normalized for the number of optional modules. Simultaneously, the number of specialization courses is the number of courses in specialization with the highest appropriate skills rate. Algorithm 4 represents the process of curricula ranking.

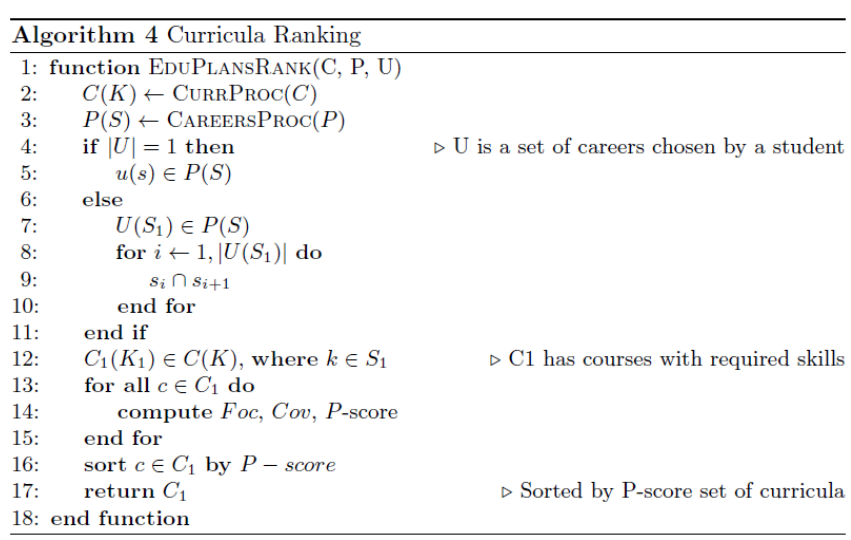

\section{RESUlTS AND Discussions}

Based on the proposed approach, a particular software service is being developed. Service contains various education process elements such as subject areas, educational programs, academic courses, and curricula. Academic courses are presented as a hierarchy of keywords. The ability to use prerequisites and results is implemented for each course, allowing to visualize its content and structure. Professions are also described using this approach. Furthermore, online courses will be represented the same way.

The service provides the university staff who oversee educational programs with an opportunity to upload keywords obtained from learning support materials; establish connections between domains and academic courses both manually and automatically and between academic courses. A keyword graph is created upon this data. The developed service is a tool for experts to create and verify connections between academic courses and other educational entities.

User interfaces enable users to effectively operate with the syllabus (Fig. 4) and provide the following features:

1) Filling in general information about the syllabus.

2) Filling in descriptions of sections and topics.

3) Describing online courses.

4) Describing assessment tools. 
5) Describing prerequisites and learning outcomes with keywords.

The last feature supports the process of educational entities' graph creation. Through connections between an academic course, its prerequisites, and learning outcomes, it is possible to build graphs showing the consistency of knowledge between the curriculum's syllabi.

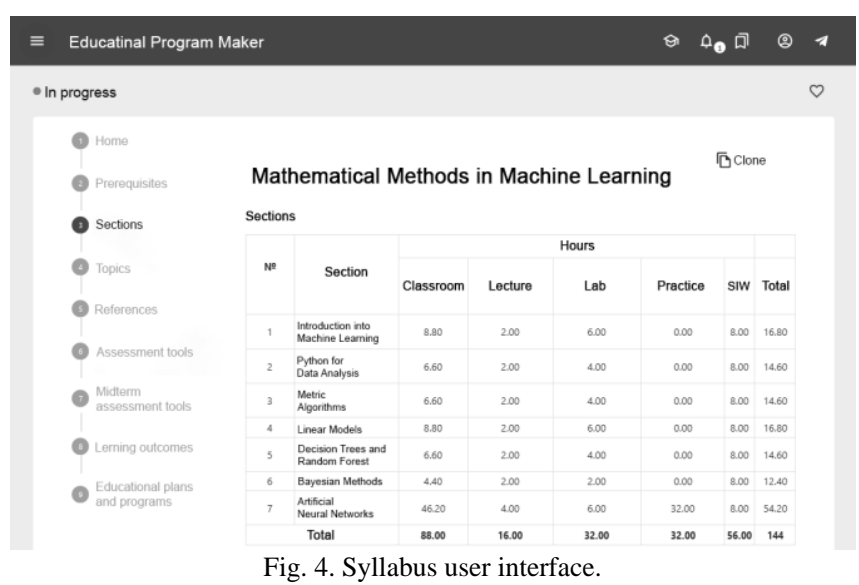

The system has corresponding interfaces to view, create, or update each educational program element. The web service may be accessed by [16]. For the individual curriculum selection, the service client must choose a profession for which, in real-time, based on relevant data, the most suitable curriculum will be selected with a choice of optional courses and specializations. Keyword configuration interfaces for describing professions are implemented in the system according to the proposed approach (Fig. 5).

Front-End Developer skills

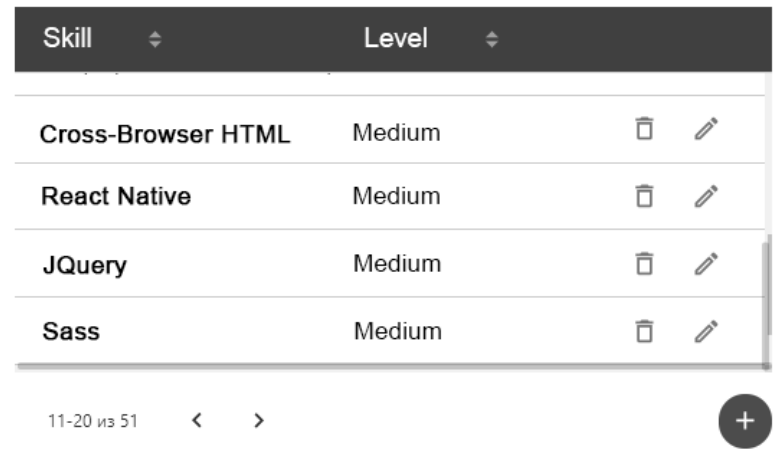

Fig. 5. Profession description customization interface.

The user gets a list of the most suitable curricula with the selection. Each of them the user can add to the personal list of curricula. There is another interface containing information about chosen curricula shown in Fig. 6.

Besides, each user can access a set of interfaces for manually building an individual learning pathway based on the chosen one. Users can independently choose optional courses and various training modules available for selection in this curriculum.

The following technologies were used. Backend services and algorithms were developed using Django 2.2, Django Rest Framework 3.10.3, and PostgreSQL 11+. React 16.13.1,
Redux 4.0.5, MaterialUI 4.9.11, and Axios 0.19 were used to build front-end interfaces. Docker 2.3.0.3 was used for continuous integration and continuous delivery. Also, various ITMO University information services were provided with API interfaces for data extraction using the Django REST framework.

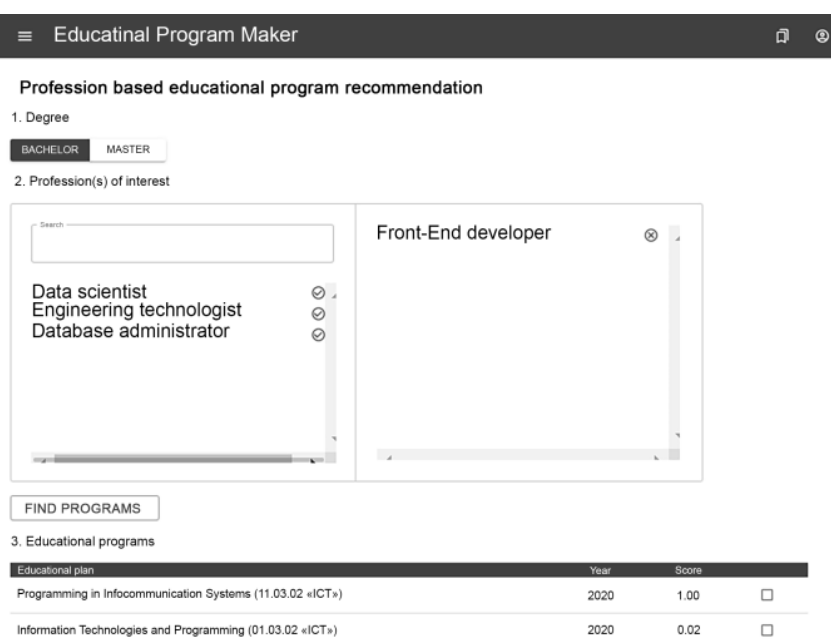

Fig. 6. Individual curricula interface.

\section{CONCLUSION}

In this study, an approach to keyword graph creation describing the relations between academic courses has been widened; and an approach to creating individual learning pathways based on target professions has been proposed.

A software service was developed for working with education process elements, which allows not only to work with various educational program elements, i.e., curricula and syllabi, but to receive recommendations to improve the quality of their materials. The service is based on the proposed approaches and satisfies the Russian State Educational Standard requirements and the concept of curriculum description. The service was tested by a team of 8 testers from the ITMO University's Department of Educational Activities. Since 24.03.2021, the service has been widely used at ITMO University, St. Petersburg, Russia.

The developed system within the proposed approach helps to solve the following objectives:

1) Ensure the development of educational program elements.

2) Provide a platform for relations between educational entities' descriptions.

3) Enable users to choose a curriculum for a specific profession.

Implementation of the software module for syllabus creation allows to describe the prerequisites and learning outcomes in a unified way and link various educational activities with controlled learning outcomes, thus disclosing academic course content.

Future work will be focused on the following tasks:

1) Develop mechanisms for collecting user interaction with educational program elements to further build a recommendation system.

2) Develop an algorithm for determining the relations between academic courses' learning outcomes and 
professional roles. Data on job descriptions from open sources will be collected as part of this algorithm. Based on the obtained data, professions will be characterized and represented as sets of keywords. Further, algorithms performing automatic matching between educational programs and professions that the graduates can acquire will be developed and implemented. Such correlations will be determined not only by a summary of the educational program but based on the keywords of all its academic courses.

3) Implement the module for the online course selection. Nowadays, educators often use online courses as a part of an academic course or as supporting material. To reduce the time consumption of the relevant course selection process, we will supply each online course with a keyword description. Moreover, several online course recommendation systems will be developed and evaluated to implement the best option as a separate module.

Additionally, the proposed approach will allow implementing algorithms for recommending online courses to a student based on his already completed courses. The system will recommend online courses that could be useful to the student practically or theoretically. The recommendation system will help individualize a student's learning pathway by advising courses meeting his area of interest.

\section{CONFLICT OF INTEREST}

The authors declare no conflict of interest.

\section{AUTHOR CONTRIBUTIONS}

A. Chernysheva proposed the methodology. A. Govorov, S. Koriakov, and V. Artamonova designed the web service and implemented the approach. S. Derkunskaia and C. Babayants tested the system and conducted the literature review. All authors participated in the writing of the paper and had approved the final version.

\section{ACKNOWLEDGMENT}

The authors would like to thank Maksim Khlopotov, an associate professor at the ITMO University's Faculty of Infocommunication Technologies, for his expertise and research commitment that led to the writing of this paper.

\section{REFERENCES}

[1] A. Govorov, A. Chernysheva, M. Khlopotov, S. Derkunskaia, and A Arzumanian, "Individual learning pathway validation based on the syllabus," in Proc. the 26th Conference of Open Innovations Association FRUCT, pp. 108-114, 2020.

[2] P. V. Sysoev, "Learning according to an individual trajectory," Language and Culture, 2013.

[3] I. M. Kharitonov, "Algorithm for the formation of the curriculum using the technique of formalized presentation of the academic discipline (on the example of the discipline "Modeling of systems")," Vestnik of Astrakhan State Technical University, vol. 2, 2011.
[4] N. Jones, "Human centered syllabus design: Positioning our students as expert end-users," Computers and Composition, vol. 49, pp. 25-35, 2018.

[5] R. Wette, "Professional knowledge in action: How experienced ESOL teachers respond to feedback from learners within syllabus and contextual constraints," System, vol. 38, no. 4, pp. 569-579, 2010.

[6] E. Dubicki, "Mapping curriculum learning outcomes to ACRL's Framework threshold concepts: A syllabus study," The Journal of Academic Librarianship, vol. 45, no. 3, pp. 288-298, 2019.

[7] A. A. Sharov, "Role of university education in the construction of professional career," Modern Pedagogy, vol. 1, 2016.

[8] A. S. Sokolova, "Career-forming education in the educational space of a modern university," Vestnik of the Moscow State Linguistic University, vol. 4, 2018.

[9] L. Khailova, "Using curriculum mapping to scaffold and equitably distribute information literacy instruction for graduate professional studies programs," The Journal of Academic Librarianship, vol. 47, no. $1,2021$.

[10] M. E. Woolley et al., "Advancing academic achievement through career relevance in the middle grades: A longitudinal evaluation of CareerStart," American Educational Research Journal, vol. 50, no. 6 , pp. 1309-1335, 2013.

[11] H. Farah et al., "Using network science to understand the link between subjects and professions," Computers in Human Behavior, vol. 106, 2020.

[12] I. Yurlovskaya, "The problem of individualization of training students in terms of the current situation," Science Studies, vol. 1, pp. 1-8, 2014

[13] E. Gregori-Giralt and J.-L. Menéndez-Varela, "The content aspect of validity in a rubric-based assessment system for course syllabuses," Studies in Educational Evaluation, vol. 68, 2021.

[14] A. Chernysheva, M. Khlopotov, and D. Zubok, "Subject area study: Keywords in scholarly article abstracts graph analysis," CEUR Workshop Proceedings, pp. 155-166, 2021.

[15] Habr Career. [Online]. Available: https://career.habr.com

[16] Educational Program maker. [Online]. Available: https://op.itmo.ru

Copyright (C) 2022 by the authors. This is an open access article distributed under the Creative Commons Attribution License which permits unrestricted use, distribution, and reproduction in any medium, provided the original work is properly cited (CC BY 4.0).

Anton Govorov received his BSc and MSc in computer science and engineering, BSc in economy and marketing from ITMO University, Saint Petersburg, Russia. He is currently a senior lecturer at the Faculty of Infocommunication Technologies and is working on his $\mathrm{PhD}$ thesis in the individualization of education

Anastasiia Chernysheva received her BSc and MSc in intelligent systems in humanities from ITMO University, Saint Petersburg, Russia, in 2019. She is currently a PhD student and an engineer at the Faculty of Infocommunication Technologies. Her research interests include natural language processing, data analysis, and learning analytics.

Svetlana Derkunskaia received her MSc in information systems and technologies from ITMO University, Saint Peterburg, Russia. She is currently a $\mathrm{PhD}$ student and an engineer at the Faculty of Infocommunication Technologies.

Valeriya Artamonova was born in 1999, Taraz, Kazakhstan. She received a BSc in intelligent systems in humanities from ITMO University, Saint Petersburg, Russia. Her current research interests include web development and machine learning.

Carina Babayants was born in March 1997. She received her BSc in intelligent systems in humanities from ITMO University, Saint Petersburg, Russia. Her research interests are in the field of data analysis.

Sergei Koriakov graduated from the Faculty of Secondary Vocational Education at ITMO University, Saint Petersburg, Russia, in 2020. He is currently an undergraduate student at the Faculty of Infocommunication Technologies. 